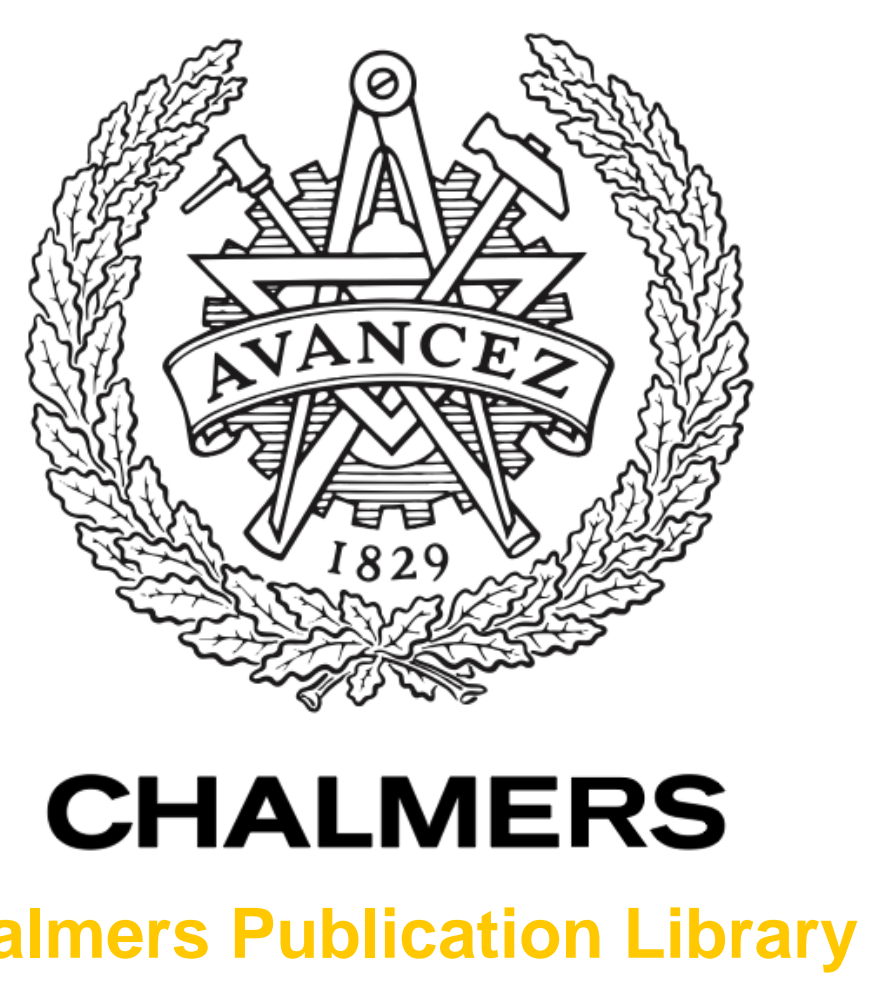

\title{
Comparison of full 3D and unit cell models for waveguide-embedded frequency multiplier arrays
}

This document has been downloaded from Chalmers Publication Library (CPL). It is the author's version of a work that was accepted for publication in:

IEEE Microwave and Wireless Components Letters (ISSN: 1531-1309)

Citation for the published paper:

Hrubó, G. ; Dahlbäck, R. ; Stake, J. (2016) "Comparison of full 3D and unit cell models for waveguide-embedded frequency multiplier arrays". IEEE Microwave and Wireless

Components Letters, vol. 26(11), pp. 954-956.

http://dx.doi.org/10.1109/LMWC.2016.2615011

Downloaded from: http://publications.lib.chalmers.se/publication/244060

Notice: Changes introduced as a result of publishing processes such as copy-editing and formatting may not be reflected in this document. For a definitive version of this work, please refer to the published source. Please note that access to the published version might require a subscription. 


\title{
Comparison of Full 3D and Unit Cell Models for Waveguide-Embedded Frequency Multiplier Arrays
}

\author{
Gergely Hrubó, Robin Dahlbäck, Student Member, IEEE, and Jan Stake, Senior Member, IEEE
}

\begin{abstract}
We present a full wave, large-signal analysis of a waveguide-embedded frequency multiplier array, solving the entire geometry using FEM EM modeling and including every nonlinear element in a Harmonic Balance simulation. The full 3D model employed provides the exact field distribution across the array, thus enabling us to account for substrate resonances, higher order mode excitation, instabilities, and diode yield. The model is compared with measurements on a $247 \mathrm{GHz}$ fixed tuned 72-diode HBV tripler. In this example, higher order mode excitation was found due to the interaction between the filter and the diode array. This can only be observed using a full 3D model, which more accurately predicts the output power versus input power and frequency. Finally, the pros and cons of a full 3D model and a unit cell model for waveguide embedded multiplier arrays are discussed.
\end{abstract}

Index Terms-Frequency multipliers, heterostructure barrier varactors, higher order modes, spatial power combining, unit cell modeling, varactors.

\section{INTRODUCTION}

$\mathbf{T}$ ODAY, solid state devices are the workhorse in high frequency electronics due to their low cost and reliability. To overcome their input power limitation, finite arrays of solid state devices using spatial power combining have been presented for amplifiers [1], oscillators [2], and frequency multipliers [3]. The arrays can be implemented in free space using lenses to guide the beam, or embedded in waveguides, resulting in a more compact component.

The unit cell model has proven accurate in free space devices, because the infinite, periodic array approximation remains valid as long as the collimated beam is densely covered by the array. However, once the array is placed in a waveguide, edge effects at the field maxima can play a significant role. Nowadays computational resources enable linear simulation of the finite array with waveguide boundary conditions using FEM modeling. Through lumped ports placed at the antennas we can include every nonlinear element in the Harmonic Balance simulation.

Several techniques have been presented to prevent different resonances in the substrates [4] [5], which together with the unit cell model can provide a fast design synthesis method. The full 3D model opens up new device analysis possibilities

Manuscript received June 29, 2016; accepted August 10, 2016. Date of publication October 25, 2016; date of current version November 4, 2016.

The authors are with the Terahertz and Millimetre Wave Laboratory, Department of Microtechnology and Nanoscience, Chalmers University of Technology, Goteborg SE-41296, Sweden (e-mail: jan.stake@chalmers.se).

Color versions of one or more of the figures in this paper are available online at http://ieeexplore.ieee.org.

Digital Object Identifier 10.1109/LMWC.2016.2615011 by enabling higher order mode excitation, diode yield, and instabilities to be accounted for. We verified our model by means of a 72 dipole coupled HBV frequency tripler array, which was designed using the unit cell method [6].

\section{MOdEL DESCRIPTIONS}

\section{A. The Device}

The evaluated frequency multiplier array consists of a $6 \times 12$ dipole coupled InGaAs HBV array with six barriers per diode and a mesa area of $20 \mu \mathrm{m}^{2}$. It is fabricated on a $185 \mu \mathrm{m}$ thick InP substrate with a bandpass filter on a $150 \mu \mathrm{m}$ thick Quartz substrate as an output matching network. A $380 \mu m$ thick InP substrate is responsible for the input matching. The output bandpass filter is a rhomb aperture frequency selective surface with $4 \times 8$ unit cells. To prevent first harmonic power leakage and present a single mode output interface, a WR10-WR3 linear taper is placed at the output. The RF measurement setup used to characterize the device is equivalent to the one presented in [6].

\section{B. Full 3D Model}

The full 3D model contains the antenna and filter arrays, input matching slab and the linear taper in a single model implemented in Ansys HFSS. The waveguide walls, antennas, and the filter were modeled with finite conductivity and lossy dielectrics were used. The mounting pocket of the slabs was not included as the model should correspond to $6 \times 12$ unit cells. All propagating higher order modes were terminated with their characteristic impedance. The Harmonic Balance simulation was carried out in NI Microwave Office with all 72 diodes present. The Chalmers HBV model [7] was used $\left(R_{s}=20 \Omega, C_{\max }=8 f F\right)$ in both the full 3D and the unit cell model, without the self-heating effect being included. DC and harmonics above the output frequency were terminated with a low impedance. A cross section of the linear part of the model is illustrated in Fig. 1.

\section{Unit cell model}

A transmission line representation of the unit cell model is shown in Fig. 2. The working principle of cascaded unit cells described in [8] was further developed by taking into account the uneven excitation powers along diodes in the different columns as well as phase variation caused by the diode nonlinearity among the unit cells. A unit cell of both the antenna array and the filter were simulated in Ansys HFSS with periodic boundary conditions. The same matching 


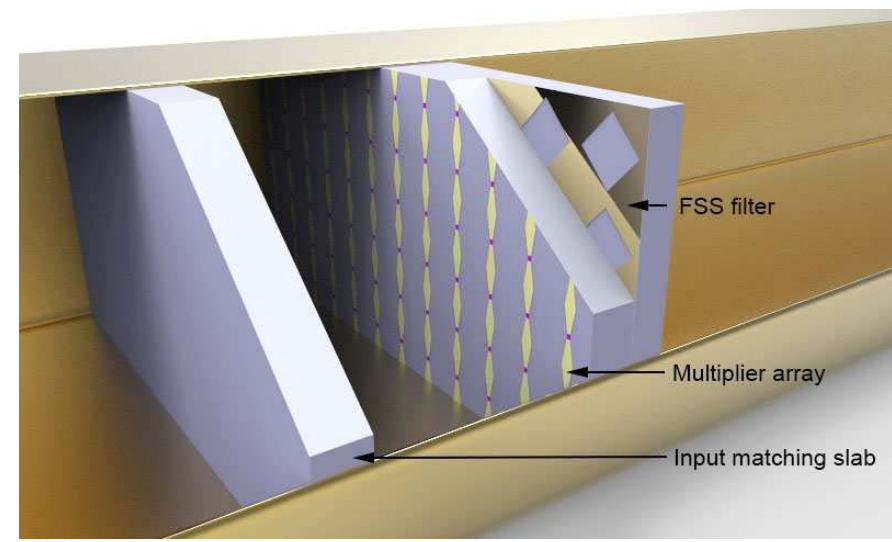

Fig. 1. Cross section of the full 3D model implemented in the 3D EM solver.

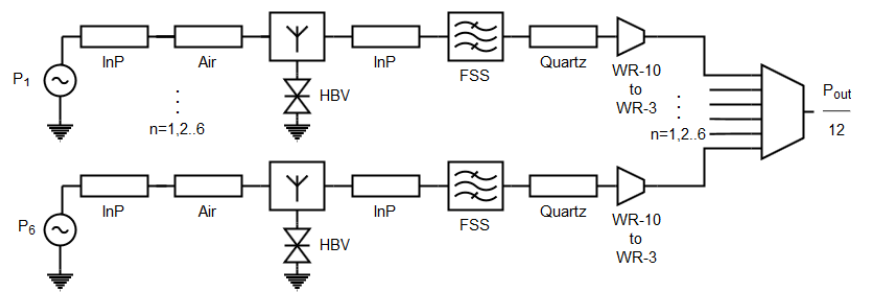

Fig. 2. Schematic of the unit cell based transmission line model. Six sets of equal unit cell chains are applied, each representing one of the six array columns modeled. The unit cell chains are excited by power corresponding to their position in the waveguide and the output is vectorially combined.

impedances as in the full 3D model were used. The following assumptions characterize our unit cell model:

1) The input power distribution matches the $\mathrm{TE}_{10}$ mode.

2) The output power only excites the $\mathrm{TE}_{10}$ mode.

3) The antenna array is infinite (no edge effect) and receives a plane wave.

4) The $\mathrm{TE}_{10}$ electrical symmetry has not been violated.

The cascaded unit cell model was simulated for 6 unit cells with a $\sin ^{2}(2 k / 13 * \pi / 2)$ for $k=1,2, \ldots 6$ power variation among them and their output power was combined with ideal power combiners.

\section{MODEL VERIFICATION}

Fig. 3 and Fig. 4 shows the frequency- and power sweep of the two models and measured data. The shaded region shows measurement uncertainty of the power meter in the selected measurement range. $2 \mathrm{~dB}$ loss was added to both models in order to account for the following unmodeled sources of errors:

- Unknown diode yield;

- Fabrication and assembling tolerances, ohmic losses in the mounting pockets;

- Losses due to surface conductivity on the array substrate originating from the imperfect removal of the seed layer used for gold plating.

The full 3D model follows the measured power sweep at the design frequency accurately, with the exception of high output powers, where thermal effects start to play an increasing role. The discrepancy between the model and measurement

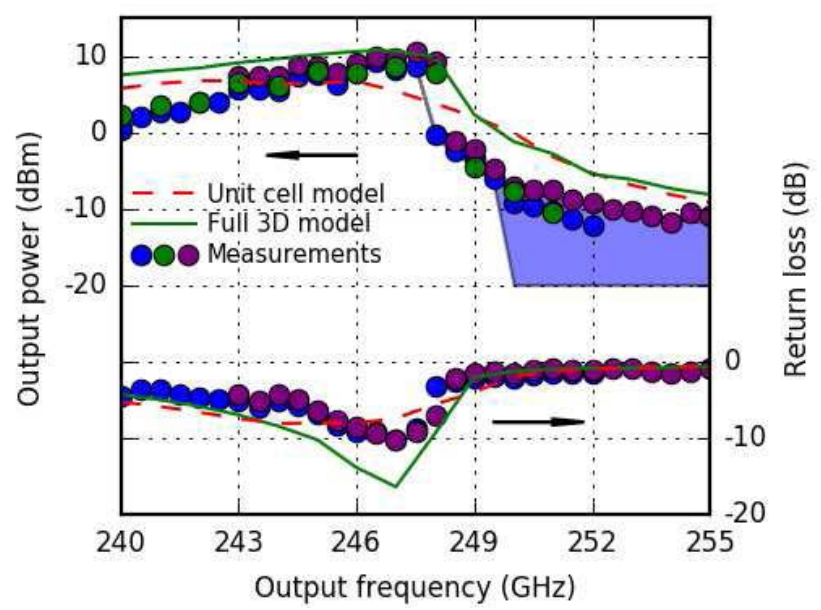

Fig. 3. Output power and return loss of three measurement runs of the same frequency multiplier array after reassembling, the full 3D model and the unit cell model as a function of output frequency for an input power of $300 \mathrm{~mW}$. The shaded region shows measurement uncertainty of the power meter in the selected measurement range with respect to the measurement denoted with blue circles.

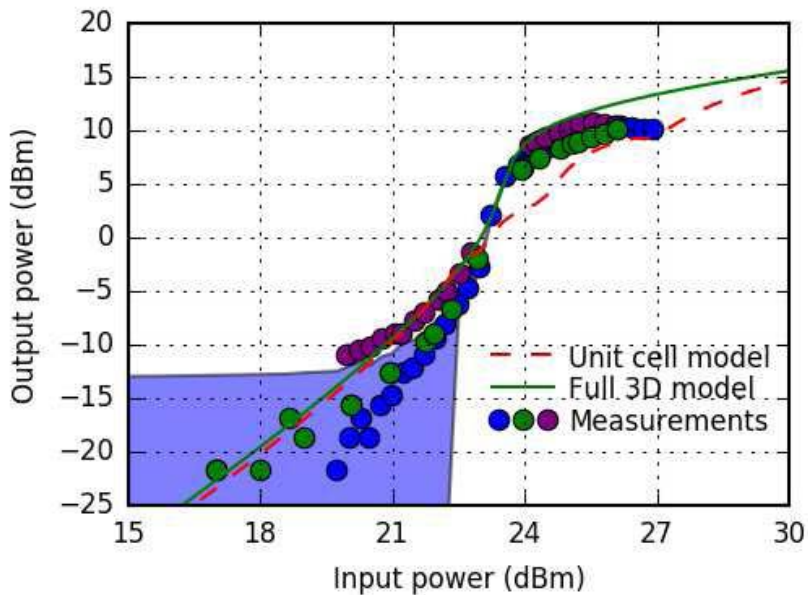

Fig. 4. Output power of three measurement runs of the same frequency multiplier array after reassembling, the full 3D model and the unit cell model as a function of input power at $247 \mathrm{GHz}$ output frequency. The shaded region shows measurement uncertainty of the power meter in the selected measurement range with respect to the measurement denoted with blue circles.

is increasing while moving away from the design frequency. A possible explanation for this is that the non-perfect diode yield narrows down the operation band. The unit cell model fails to follow the abrupt changes of the measured curve - which is due to the interaction between the nonlinear elements - and thus peaks at a $2 \%$ shifted frequency.

\section{RESUlTS AND Discussion}

Voltage and current meters placed in the full 3D model's Harmonic Balance simulation provide a qualitative overview of the assumptions listed above. Fig. 5 shows the incident power and phase difference at the fundamental frequency among the diodes located in different columns of the array. There is a maximum normalized amplitude deviation of 0.15 and a maximum phase deviation of 1 degree between the two models. 


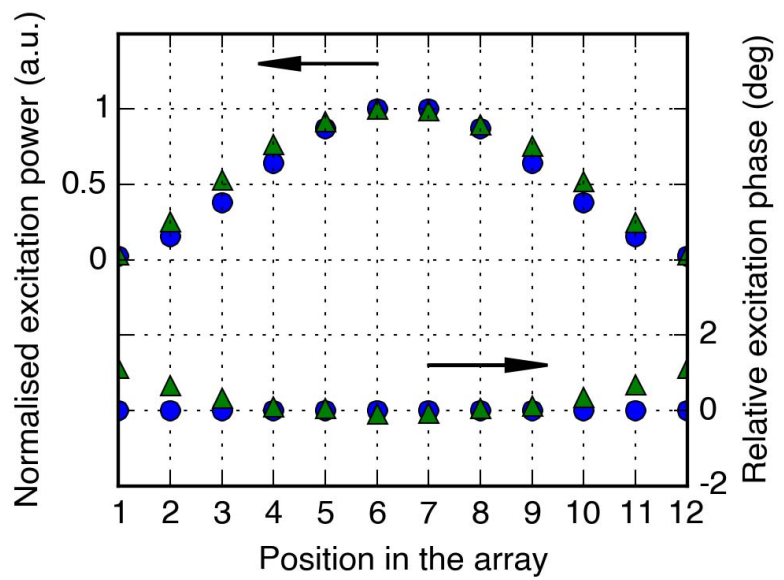

Fig. 5. Input power and phase distribution among different columns of the array: unit cell assumption (circles) and full 3D model (triangles).

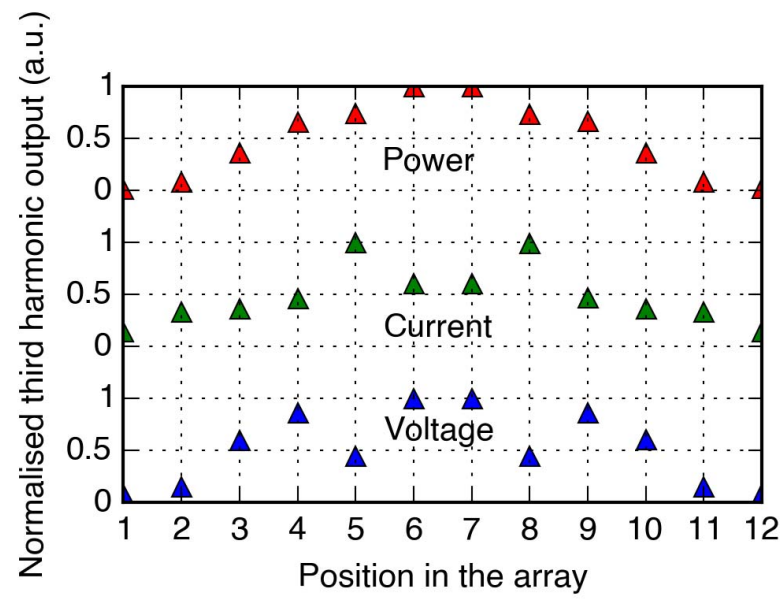

Fig. 6. Magnitude distribution of the third harmonic power, current and voltage of the diodes in different columns for $300 \mathrm{~mW}$ input power at $247 \mathrm{GHz}$ output frequency in the full 3D model. The power distribution deviates from the pure fundamental waveguide mode due to the abrupt changes in the voltage and current.

Fig. 6 shows the voltage, current and power distribution at the output frequency in different columns. Current maxima and voltage minima appear in columns number 2, 5, 8, and 11, which cause the output power to deviate from that of a fundamental mode. This is depicted in Fig. 7, where the magnitude of the electric field is plotted in a horizontal cross section of the full 3D simulation. A standing wave between columns 2, 5,8 and 11 , and the filter surface can be observed due to the fact that the FSS-filter is not treated as a homogeneous surface by the antenna array. Substrate thickness being approximately half the guided wavelength and having the critical columns not overlapping with the filter unit cell introduces a short circuit for the corresponding diodes. This example shows that even though higher order mode excitation due to the diode nonlinearity (as a result of non-uniform conversion efficiency across the array) can be predicted by the unit cell method [3], the linear electromagnetic environment must also be considered when aiming for single-mode excitation. This is, by definition, beyond the limits of the unit cell model and can only be revealed using full 3D model.

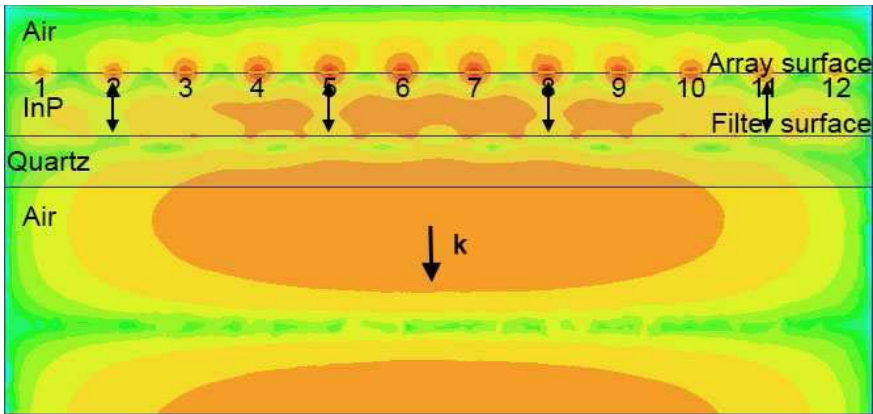

Fig. 7. Magnitude of the electric field in a top view cross section, showing the interaction between the varactor array and the filter. Arrows indicate the standing waves between certain columns of the multiplier array and the filter.

TABLE I

NUMBER OF TETRAHEDRONS REQUIRED PER FREQUENCY POINT FOR THE SIMULATION OF THE LINEAR PART OF BOTH MODELS.

\begin{tabular}{|l|l|l|}
\hline & Unit cell model & Full 3D model \\
\hline Fundamental frequency & $7.4^{*} 10^{3}$ & $3.6^{*} 10^{5}$ \\
\hline Output frequency & $2.0^{*} 10^{4}$ & $1.2^{*} 10^{6}$ \\
\hline
\end{tabular}

Finally, Table 1 summarizes the total computational resource required for both models at each frequency point.

\section{CONCLUSION}

A unit cell and a full 3D model has been compared for a waveguide-embedded frequency multiplier array. The full 3D model better predicts the output power as a function of frequency and input power. Our suggested design methodology is a preliminary unit cell design that is then fine tuned and further improved using a full 3D model. To the best of our knowledge this is the first presentation of a complete 3D-EM simulation where all the nonlinear elements of a waveguideembedded frequency multiplier array have been included in a Harmonic Balance simulation.

\section{REFERENCES}

[1] M. Kim et al., "A 100-element HBT grid amplifier," IEEE Trans. Microw. Theory Techn., vol. 41, no. 10, pp. 1762-1771, Oct. 1993.

[2] J. Bae, M. Fujita, and K. Mizuno, "A W-band overmoded-waveguide oscillator with Gunn diodes," IEEE Trans. Microw. Theory Techn., vol. 49, no. 12, pp. 2554-2559, Dec. 2001.

[3] S. A. Rosenau, "Quasi-optical overmoded waveguide frequency multiplier grid arrays," Ph.D. dissertation, Univ. California Davis, Davis, CA, USA, 2001.

[4] A. Al-Zayed, R. R. Swisher, F. Lecuyer, A. C. Guyette, Q. Sun, and M. P. D. Lisio, "Reduction of substrate-mode effects in powercombining arrays," IEEE Trans. Microw. Theory Techn., vol. 49, no. 6, pp. 1067-1072, Jun. 2001.

[5] M. A. Morgan and S. K. Pan, "Graphical prediction of trapped mode resonances in sub-mm and THz waveguide networks," IEEE Trans. THz Sci. Technol., vol. 3, no. 1, pp. 72-80, Jan. 2013.

[6] R. Dahlbäck, J. Vukusic, R. M. Weikle, II, and J. Stake, "A tunable 240-290 GHz waveguide enclosed 2-D grid HBV frequency tripler," IEEE Trans. THz Sci. Technol., vol. 6, no. 3, pp. 503-509, May 2016.

[7] M. Ingvarson, J. Vukusic, A. O. Olsen, T. A. Emadi, and J. Stake, "An electro-thermal HBV model," in IEEE MTT-S Int. Dig., Jun. 2005, p. 3.

[8] W. A. Shiroma, S. C. Bundy, S. Hollung, B. D. Bauernfeind, and Z. B. Popovic, "Cascaded active and passive quasi-optical grids," IEEE Trans. Microw. Theory Techn., vol. 43, no. 12, pp. 2904-2909, Dec. 1995. 